\title{
A note on $x Q x$ as a modelling and solution framework for the Linear Ordering Problem
}

\section{Mark Lewis}

Craig School of Business,

Missouri Western State University,

4525 Downs Dr., Saint Joseph, MO, 64507, USA

E-mail: mlewis14@missouriwestern.edu

\section{Bahram Alidaee}

School of Business, Holman Hall,

University of Mississippi,

University, MS, 38677, USA

E-mail: balidaee@bus.olemiss.edu

\section{Fred Glover}

Leeds School of Business and

Department of Electrical and Computer Engineering,

University of Colorado,

Boulder, CO, 80309, USA

E-mail: fred.glover@colorado.edu

\section{Gary Kochenberger*}

School of Business,

University of Colorado,

1250 14th St., Denver, CO, 80202, USA

E-mail: Gary.kochenberger@cudenver.edu

${ }^{*}$ Corresponding author

\begin{abstract}
This paper expands the list of $0-1$ problems that can be effectively modelled and solved as Unconstrained Quadratic Binary Programs (UQPs). UQP has been presented as a general-purpose modelling approach with application to a broad range of problem classes (Kochenberger et al., 2004). In this paper, we demonstrate that the Linear Ordering Problem (LOP) can be easily recast so that it can be treated as a UQP problem, and that large instances of the LOP can be effectively handled within this framework. Computational results are given demonstrating the viability and attractiveness of this approach.
\end{abstract}

Keywords: linear ordering; integer programming; metaheuristics.

Reference to this paper should be made as follows: Lewis, M., Alidaee, B., Glover, F. and Kochenberger, G. (2009) 'A note on xQx as a modelling and solution framework for the Linear Ordering Problem', Int. J. Operational Research, Vol. 5, No. 2, pp.152-162. 
Biographical notes: Mark Lewis has a BS in Electrical Engineering from the University of Kansas and has worked as an engineer in avionic development at Lockheed Martin for 12 years before earning his $\mathrm{PhD}$ in Operations Research from Southern Methodist University in Dallas, Texas, USA, in 2000. His interests include mathematical modelling and algorithm development and implementation. He has published in INFORMS Journal on Computing, OR Letters, Computers and Operations Research, International Journal of Operations and Quantitative Management and others. He is currently an Associate Professor of Information Systems and Operations Management at Missouri Western State University.

Bahram Alidaee received his BS from the University of Tehran, Iran, his MBA from the University of North Texas and his PhD from the University of Texas at Arlington. He is currently a Professor of Operations Management at the School of Business Administration, the University of Mississippi. His research interests include optimisation, heuristic programming, complex systems, and game theory. He has published in variety of journals. $\mathrm{He}$ is a member of INFORMS, ICS, DSI, POMS, APICS, ISM, IEEE Computer Society.

Fred Glover is a Distinguished Professor of the University of Colorado System. He has authored or coauthored more than 350 published papers and eight books in the fields of mathematical optimisation, Computer Science and artificial intelligence, with particular emphasis on practical applications in industry and government. He is the recipient of the von Neumann Theory Prize, a member of the National Academy of Engineering, and has received numerous other awards and honourary fellowships. He serves on the advisory boards of several organisations and is cofounder of OptTek Systems, Inc.

Gary Kochenberger's academic specialty concerns Building, Testing, and Implementing Algorithms for solving resource allocation problems that arise in both the private and public sectors. In recent years, his focus has been on problems of a combinatorial nature. He has published four books and numerous research papers in such journals as the Operational Research, Management Science, Mathematical Programming, Journal of Optimisation Theory and Applications, Operation Research, Computers and Operations Research, Naval Research Logistics Quarterly, Decision Sciences, Journal of the Operational Research Society, European Journal of OR, Interfaces, Operations Research Letters, Omega, and the Journal of the Production and Operations Management Society.

\section{Introduction}

The Linear Ordering Problem (LOP) is well known for its diversity of application as well as its computational challenge. This important problem, which is known to be NP-hard, has attracted considerable attention in the literature resulting in various efficient, tailored solution approaches. In contrast to such special-purpose approaches, this paper highlights the potential application of a general-purpose modelling and solution approach to LOP. Specifically, we indicate how the unified framework for combinatorial optimisation problems given by the Unconstrained Quadratic Binary Program (UQP)

UQP: $\max x^{t} Q x \quad x \in\{0,1\}$ 
can be employed to model and solve large instances of LOP. We present a simple transformation enabling LOP to be modelled as an instance of UQP and illustrate the potential viability of this approach by preliminary computational testing.

In a theoretical sense, the UQP formulation is as general as the $0-1$ pure Integer Linear Programming (ILP) problem, and hence it becomes valuable to know the range of $0-1$ ILP problems for which UQP also yields an exploitable design. In particular, we are interested in discovering those $0-1$ problems whose UQP formulation gives a basis for applying methods that efficiently yield high-quality solutions. From this standpoint, we are motivated to compare the UQP model against classical 0-1 ILP models, applied to problems from different settings to determine which problems are more exploitable by UQP and which by $0-1$ ILP. Thus, in this paper, we illustrate how, from the standpoint of being a general-purpose model design applicable to many other kinds of problems, UQP is quite effective at solving LOP.

We focus on the LOP problem as one that is challenging and has a significant range of applications, and compare the use of the UQP model solved by a Tabu search method (which has been shown to be effective for this formulation) against the use of the $0-1$ ILP model solved by CPLEX (a leading method based on linear programming using branch-and-cut and more recently incorporating some heuristics). It is noted that CPLEX is an exact optimising method, guaranteeing an optimal solution in a finite (though possibly large) time, while Tabu search is a heuristic approach that does not offer such a guarantee. However, we know of no general-purpose heuristic for the 0-1 ILP formulation that dominates CPLEX over a notable range of problems. This is due in part to the fact that CPLEX has been progressively modified over the years by teams of skilled researchers to incorporate a variety of components that may appropriately be considered heuristic. For the restricted goal of finding a first-feasible solution, the feasibility pump method of Fischetti et al. (2005) has proved better than CPLEX, but cannot compete with CPLEX for finding optimal or near-optimal solutions.

It is on the dimension of quickly finding elite (i.e., very high quality) solutions that we seek to compare the UQP formulation to the $0-1$ ILP formulation, and for this purpose, CPLEX is among the best methods available. Our previous findings of the power of the UQP formulation on a wide range of other applications provides impetus for investigating whether it may also prove equally effective for the LOP problem.

Applications of the linear ordering model have been reported in a wide variety of problem settings, including economics, logistics, social sciences, and discrete mathematics (e.g., see Bruyèrea and Carton, 2007; Mitchell and Borchers, 2000; Steiner and Stephenson, 2000; Laguna et al., 1999; Mitchell et al., 1998). The most oft-quoted example is from economics where the problem concerns the triangulation of input-output matrices - as seen in Grötschel et al. (1984), Mitchell and Borchers (2000). Linear ordering applications often involve the ranking of pair-wise comparisons such as used in analytical hierarchical processing, with LOP providing an alternative method of maximising consistency between comparisons (see Laguna et al., 1999). Applications in bioinformatics are seen in Bar-Joseph et al. (2001) and Biedl et al. (2001), which discuss the optimal ordering of leaves in binary trees resulting from the hierarchical clustering of genes. A potential application in website design maximises an ordering of pages based on clickstream or shopping cart data, see Cooley (2003). 


\section{Mathematical model}

Given an $n \times n$ matrix of weights $C=\left\{c_{i j}\right\}$, we wish to find the permutation of rows and columns of $C$ such that the sum of the weights of the upper triangular matrix are maximised. Letting $x_{i j}=1$ if item $i$ precedes item $j$ in the ordering, the standard binary integer programming model (see Grötschel et al., 1984) for LOP is:

$$
\begin{aligned}
& \text { LO_IP: Max } \sum_{i<j}^{n} c_{i j} x_{i j}+\sum_{j<i}^{n} c_{i j}\left(1-x_{j i}\right) \\
& \text { s.t. } x_{i j}+x_{j k}-x_{i k} \leq 1 \quad \forall(i, j, k): i<j<k \\
& x_{i j}+x_{j k}-x_{i k} \geq 0 \quad \forall(i, j, k): i<j<k \\
& x_{i j} \in\{0,1\} \quad \forall(i, j): i<j \text {. }
\end{aligned}
$$

\subsection{Solving $L O P$}

Exact methods for solving LOP, which concentrate on LP-based methods and facet generation, can be found in Grötschel et al. (1984) and Mitchell and Borchers (2000). However, because of the computational difficulty in solving LOP to proven optimality, various heuristic methods have been proposed for this problem. The most notable ones are the scatter search approaches found in Laguna and Martí (2003), Laguna et al. (1999), the Lagrangian-based approach given in Belloni and Lucena (2003) and the genetic hill-climbing approach to linear arrangements of undirected graphs in Poranen (2005). All these methods are reported to perform well on medium- to large-scale problems. Rao and Richa (2004) developed a recursive polynomial time $O(\log n)$ approximation algorithm for the minimum linear arrangement problem for a general graph with $n$ nodes. An $\alpha$-approximation algorithm finds a solution that is at most $\alpha$ times the cost of an optimal solution.

It is to be emphasised that the solution approaches highlighted above are specifically designed for LOPs, while the approach we take in this paper is fundamentally different. Rather than an attempt to solve LOP as a large 0-1 linear integer program or create specialised heuristics with tailored data structures, we re-cast and solve LOP as an instance of the general UQP model. Recent papers (see Kochenberger et al., 2004, 2005; Lewis et al., 2005) have reported on the successful application of this unified framework for a variety of problems including vertex colouring and task allocation problems.

With the unified approach, problems are re-cast into the common modelling form of UQP. This problem, in turn, is solved by a solution method designed not for the original problem structure but for the generic UQP model, so that a single optimiser, i.e., one designed to solve UQP, may be used to solve a wide variety of combinatorial problems. Our main objective in this paper is to illustrate how this approach can be easily and successfully employed to solve the LOP.

The transformation from a given mathematical structure into the unified framework of UQP is accomplished by imposing quadratic infeasibility penalties for violating the original problem constraints. This approach can, in principle, be applied to any problem with linear constraints, bounded integer variables, and a linear or quadratic objective function. In this regard, we refer to a penalty function $g(x)$ as being a 
Valid Infeasibility Penalty (VIP) if $g(x)$ is zero when $x$ is feasible and positive otherwise. Further, if by the selection of an appropriate positive scalar penalty $P$, the combined objective function (i.e., including the penalties) is optimised over binary $x$ when $g(x)=0$, then we call the term $P g(x)$ a Sufficient Infeasible Penalty (SIP). (The minus sign in the combined objective function is of course replaced by a plus sign if the goal is one of minimisation.) For models with linear constraints, it is always possible to find quadratic $V I P s$ and associated penalties $P$ to yield corresponding SIPs, and thus the constrained problem can be re-cast into the form of UQP by identifying the appropriate $Q$ matrix so that $x^{t} Q x$ represents the combined function that includes the $P g(x)$ term. Generally, VIPs will not be known and will have to be discovered. Such discovery is straightforward as outlined in Boros and Hammer (2002), Hammer and Rudeanu (1968), and Hansen (1970). Although for certain problem classes, such as LOP, VIPs are known in advance, making the re-casting into the form of UQP even easier. For example, refer to the constraints (2) and (3) and consider the penalty function

$$
g(x)=g\left(x_{i j}, x_{j k}, x_{i k}\right)=\left(x_{i k}+x_{i j} x_{j k}-x_{i j} x_{i k}-x_{j k} x_{i k}\right) .
$$

Examining the truth table for $x_{i j}, x_{j k}$ and $x_{i k}$ (see Table 1) reveals that $g(x)$ is a VIP since it is zero for $x$ satisfying (2) and (3) and otherwise positive, i.e., $g(1,1,0)$ violates equations (2) and $g(0,0,1)$ violates equations (3).

Table 1 Truth table for penalty function $g(x)$

\begin{tabular}{lccc}
\hline$x_{i j}$ & $x_{j k}$ & $x_{i k}$ & $g(x)$ \\
\hline 0 & 0 & 0 & 0 \\
0 & 0 & 1 & 1 \\
0 & 1 & 0 & 0 \\
0 & 1 & 1 & 0 \\
1 & 0 & 0 & 0 \\
1 & 0 & 1 & 0 \\
1 & 1 & 0 & 1 \\
1 & 1 & 1 & 0 \\
\hline
\end{tabular}

Thus, by identifying an appropriate positive penalty $P$ and subtracting $P g(x)$ from the objective function of equation (1) for each $i<j<k$, LO_IP is directly recast into the form of

$$
\begin{aligned}
& \text { LO_UQP : } \max x^{t} Q x \\
& x \text { binary }
\end{aligned}
$$

where $x$ is a vector with $n^{\prime}=n(n-1) / 2$ variables and $Q$ is an $n^{\prime} \times n^{\prime}$ symmetric matrix.

Note that in the recasting of LO_IP into LO_UQP, we go from a linear model with many constraints to a quadratic model with no constraints and the transformation of LO_IP into LO_UQP is accomplished without the introduction of new variables. Also, the original objective function information for LO_IP appears on the main diagonal of $Q$, with the scalar penalty, $P$, sufficiently large so that solutions to LO_UQP are feasible for LO_IP. In the computational testing reported in Section 3, choosing $P=20$ (twice the maximum $c_{i j}$ value) was sufficient in that our solutions were feasible 
in all cases. Additional testing indicated that a wide range of $P$ values has very little effect on the results.

\subsection{Example}

The procedure given above is illustrated by the following example found in Laguna et al. (1999). Consider the initial table of weights

\begin{tabular}{l|llll}
\multicolumn{1}{c}{} & 1 & 2 & 3 & 4 \\
\cline { 2 - 5 } 2 & 0 & 12 & 5 & 3 \\
3 & 4 & 0 & 2 & 6 \\
4 & 8 & 3 & 0 & 9 \\
4 & 11 & 4 & 2 & 0
\end{tabular}

where the initial permutation is $p=(1,2,3,4)$ with value 37 .

The LO_IP model is:

$$
\begin{array}{lll}
\operatorname{Max} & x_{0}=32+8 x_{12}-3 x_{13}-8 x_{14}-1 x_{23}+2 x_{24}+7 x_{34} \\
\text { s.t. } & x_{12}+x_{23}-x_{13} \leq 1 & x_{12}+x_{23}-x_{13} \geq 0 \\
& x_{12}+x_{24}-x_{14} \leq 1 & x_{12}+x_{24}-x_{14} \geq 0 \\
& x_{13}+x_{34}-x_{14} \leq 1 & x_{13}+x_{34}-x_{14} \geq 0 \\
& x_{23}+x_{34}-x_{24} \leq 1 & x_{23}+x_{34}-x_{24} \geq 0
\end{array}
$$

Utilising the penalties given by equation (5), the equivalent LO_UQP model is completely specified by the $6 \times 6 Q$ matrix:

$$
Q=\left[\begin{array}{cccccc}
8 & P & P & -P & -P & 0 \\
P & -3-2 P & P & P & 0 & -P \\
P & P & -8-4 P & 0 & P & P \\
-P & P & 0 & -1 & P & -P \\
-P & 0 & P & P & -2-2 P & P \\
0 & -P & P & -P & P & 7
\end{array}\right] .
$$

Choosing the scalar penalty, $P$, to be ten and solving the problem:

$$
\operatorname{Max} x_{0}=32+x^{t} Q x
$$

yields the value 15 for $x^{t} Q x$ with $x_{12}$ and $x_{34}=1$ (all other variables equal to zero). Thus, the optimum objective function value is $15+32=47$ and the corresponding permutation is: $p=(3,4,1,2)$ yielding the table shown below. 


\begin{tabular}{c|cccc}
\multicolumn{1}{c}{} & 3 & 4 & 1 & 2 \\
\cline { 2 - 5 } 3 & 0 & 9 & 8 & 3 \\
4 & 2 & 0 & 11 & 4 \\
1 & 5 & 3 & 0 & 12 \\
2 & 2 & 6 & 4 & 0
\end{tabular}

\section{Computational experience}

To test the UQP approach to LOP, we randomly generated a set of linear ordering test problems. The publicly available test problems provided by LOLIB (1997) are known to be fairly easy to solve exactly with a commercial solver, as reported in Belloni and Lucena (2003) and as a result, several authors (Belloni and Lucena, 2003; Mitchell and Borchers, 2000) have proposed problem generators intended to produce difficult instances of LOP. In generating the problems considered here, we followed the approach used in Mitchell and Borchers (2000) to create dense, difficult problems. The main diagonal elements of the generated weight matrix $C$ are zero while the off-diagonal elements are randomly selected from a uniform distribution between zero and ten. Because almost every pair of distinct elements in $C$ has non-zero weights, these problems are very dense. As shown below, these problems proved to be difficult for CPLEX, even when set to emphasise quickly finding feasible integer solutions.

Table 2 describes the basic characteristics of the 12 problems generated. Note that the third column of the table gives the number of variables in both the LO_UQP and LO_IP models. However, the fourth column applies only to LO_IP as the quadratic model (LO_UQP) is unconstrained except for the binary restrictions.

Table 2 Problem characteristics

\begin{tabular}{lrcr}
\hline Problem ID & $n$ & \# variables & \# constraints \\
\hline LO_LAK_20 & 20 & 190 & 2280 \\
LO_LAK_30 & 30 & 435 & 8,120 \\
LO_LAK_40 & 40 & 780 & 19,760 \\
LO_LAK_50 & 50 & 1225 & 39,200 \\
LO_LAK_60 & 60 & 1770 & 68,440 \\
LO_LAK_70 & 70 & 2415 & 109,480 \\
LO_LAK_80 & 80 & 3160 & 164,320 \\
LO_LAK_90 & 90 & 4005 & 234,960 \\
LO_LAK_100 & 100 & 4950 & 323,400 \\
LO_LAK_150 & 150 & 11175 & $1,102,600$ \\
LO_LAK_200 & 200 & 19900 & $2,626,800$ \\
\hline
\end{tabular}

Each of the test problems was re-cast into LO_UQP and solved by a basic Multi-Start Tabu search with path Re-linking heuristic (called MSTR), described in Lewis (2005). The basic move structure in MSTR is defined by a simple one-opt method 
(see Palubeckis, 2004) with an additional set of elite solutions, for re-linking and re-starts, maintained as the search process unfolds. When no improvements can be found by flipping the value of a single binary variable, the multi-start approach perturbs one of the elite solutions by setting those variables that were most often at a level of one to zero (and vice versa) and giving them longer tabu tenure. As an intensification strategy, path re-linking is applied periodically between the current solution and the elite set as well as to new incumbent solutions. In all cases, the results obtained, as given in the following section, were verified as feasible with respect to the original constrained LO_IP formulation.

\subsection{Results of testing}

All problems were run using a 3.2 GHz Dell OptiPlex with $1 \mathrm{~GB}$ of RAM. To provide a comparison between two general-purpose and one tailored approach, we tested a range of problems using MSTR, CPLEX v9.1 and Scatter Search. CPLEX is an industry standard branch-and-cut Mixed Integer Program solver that performs very well on smaller LOPs via its solid foundation in linear programs, as well as heuristics. Scatter Search's feasible-move permutations with LOP-tailored data structures (see Laguna and Marti, 2003) are included to gauge the quality of the MSTR solutions for the larger problems unsolvable by CPLEX.

CPLEX has numerous parameters to aid in solving or quickly finding quality solutions. Because the problem grows quickly in size, CPLEX branch-and-cut explores fewer and fewer nodes of the search tree as size increases. To partially address this issue and for a more thorough comparison to our general-purpose heuristic, we investigated the option of setting the user-specified parameters for CPLEX to emphasise quickly finding integer feasible solutions (as opposed to the more time-consuming tasks of proving optimality). This, however, resulted in little improvement over default settings. Two other CPLEX parameters that help with large, difficult integer problems are: Relaxation-Induced Neighbourhood Search, which heuristically explores the neighbourhood of the current solution and preprocessing relaxation, which performs preprocessing on the IP relaxation (no preprocessing reductions can be made on the IP). Table 3 illustrates the beneficial effects of these two parameters for larger problem sizes, with the average improvement being $0.8 \%$. There were no synergistic improvements by combining the parameter settings.

Table 4 presents the results from solving the 12 LO_LAK problems with Scatter Search, MSTR and the best CPLEX solution. The first three problem solutions are known to be optimal. The table also illustrates the general range of problem sizes solvable by CPLEX and MSTR. For $n>80$, CPLEX was unable to find feasible integer solutions within the $1 \mathrm{~h}$ time frame, while MSTR stays within $95 \%$ of the best-known solutions for all problems. Although larger problem instances can be solved with our current approach, a performance decrease was noted on the two largest problems and instances with $n$ greater than 200 would benefit from different data structures or larger amounts of computer memory. 
Table 3 CPLEX solutions comparison using various parameters

\begin{tabular}{lcccccc}
\hline & \multicolumn{2}{c}{$\begin{array}{c}\text { Emphasise integer } \\
\text { feasibility }\end{array}$} & \multicolumn{2}{c}{$\begin{array}{c}\text { Relaxation Induced Neighbourhood } \\
\text { Search (RINS) }\end{array}$} & $\begin{array}{c}\text { Preprocessing of } \\
\text { IP relaxation }\end{array}$ \\
\cline { 2 - 7 } Problem ID & 10 min & 1 hour & 10 min & 1 hour & 10 min & 1 hour \\
\hline LAK_20 & 1140 & 1140 & 1140 & 1140 & 1140 & 1140 \\
LAK_30 & 2520 & 2520 & 2520 & 2520 & 2520 & 2520 \\
LAK_40 & 4475 & 4480 & 4475 & 4480 & 4475 & 4480 \\
LAK_50 & 6731 & 6903 & 6731 & 6949 & 6919 & 6919 \\
LAK_60 & 9655 & 9779 & - & 10054 & 9765 & 9765 \\
LAK_70 & - & 13140 & - & 12992 & - & 13198 \\
LAK_80 & - & - & - & 16993 & - & 16890 \\
LAK_90 & - & - & - & - & - & - \\
LAK_100 & - & - & - & - & - & - \\
LAK_150 & - & - & - & - & - & - \\
LAK_200 & - & - & - & - & - & - \\
\hline
\end{tabular}

Table 4 SS/CPLEX/MSTR solution comparison

\begin{tabular}{lccccc}
\hline & & \multicolumn{3}{c}{ CPLEX } & \multicolumn{3}{c}{ MSTR } \\
\cline { 3 - 6 } Problem ID & Scatter search & 10 min & 1 hour & 10 min & 1 hour \\
\hline LAK_20 & 1140 & 1140 & 1140 & 1140 & 1140 \\
LAK_30 & 2520 & 2520 & 2520 & 2520 & 2520 \\
LAK_40 & 4480 & 4475 & 4480 & 4469 & 4469 \\
LAK_50 & 6997 & 6919 & 6949 & 6963 & 6963 \\
LAK_60 & 10139 & 9765 & 10054 & 10042 & 10064 \\
LAK_70 & 13630 & - & 13198 & 13400 & 13416 \\
LAK_80 & 17788 & - & 16993 & 17276 & 17331 \\
LAK_90 & 22490 & - & - & 21921 & 22179 \\
LAK_100 & 27591 & - & - & 26667 & 27031 \\
LAK_150 & 61414 & - & - & 57944 & 59414 \\
LAK_200 & 107829 & - & - & 100551 & 103031 \\
\hline
\end{tabular}

\section{Summary and conclusions}

In this paper, we presented a simple and easy-to-implement transformation that enables the standard linear $0-1$ model of the LOP to be re-cast into the form of an unconstrained UQP. In doing so, one goes from a linear model with many constraints to a quadratic model with no constraints. In addition, we demonstrated that basic metaheuristic methods, like the tabu search approach utilised here, can be used to efficiently generate high-quality solutions for LOP once this problem is put in the form of UQP. Note that the solution method employed here was very basic and was designed for the general instance of UQP. High-quality solutions were efficiently obtained by this 
modelling/solution framework with no specialisation at all for the class of problems being solved.

The results reported in this paper are consistent with the competitive results obtained by the UQP approach to other problem classes (see, for instance Kochenberger et al., 2005; Lewis et al., 2005). Our performance here serves to further emphasise the viable, robust nature of UQP as a fruitful modelling and solution framework for various combinatorial optimisation problems. As new and improved optimisers become available for UQP, this unified framework will correspondingly become an increasingly attractive approach for rapidly finding high-quality solutions to these difficult problems.

\section{Acknowledgement}

We thank the anonymous reviewers for their careful attention to detail and helpful comments.

\section{References}

Bar-Joseph, Z., Gifford, D.K. and Jaakkola, T.S. (2001) 'Fast optimal leaf ordering for hierarchical clustering'. Bioinformatics, Vol. 17, pp.S22-S29.

Belloni, A. and Lucena, A. (2003) 'Lagrangian heuristics for the linear ordering problem', Metaheuristics: Computer Decision-Making, Kluwer Academic Publishing, Norwell, MA, pp.37-63.

Biedl, T., Brejova, B., Demaine, E., Hamel, A. and Vinar, T. (2001) Optimal Arrangement of Leaves in the Tree Representing Hierarchical Clustering of Gene Expression Data, Technical Report 2001-14, University of Waterloo, Canada.

Boros, E. and Hammer, P.L. (2002) 'Pseudo-Boolean optimization', Discrete Allied Math., Vol. 123, Nos. 1-3, pp.155-225.

Bruyèrea, V. and Carton, O. (2007) 'Automata on linear orderings', Journal of Computer and System Sciences, Vol. 73, No. 1, pp.1-24.

Cooley, R. (2003) 'The use of web structure and content to identify subjectively interesting web usage patterns', ACM Transactions on Internet Technology, Vol. 3, No. 2, pp.93-116.

Fischetti, M., Lodi, A. and Glover, F. (2005) 'The feasibility pump', Mathematical Programming - Series A, Vol. 104, No. 1, pp.91-104

Grötschel, M., Jünger, M. and Reinelt, G. (1984) 'A cutting plane algorithm for the linear ordering problem’, Operations Research, Vol. 32, No. 6, pp.1195-1220.

Hammer, P. and Rudeanu, S. (Eds.) (1968) Boolean Methods in or and Related Areas, Springer-Verlag, Berlin, Heidelberg, New York.

Hansen, P. (1970) 'Methods of nonlinear 0-1 programming', Annals of Discrete Math., Vol. 5, pp.53-70.

Kochenberger, G., Glover, F., Alidaee, B. and Rego, C. (2005) 'An unconstrained binary programming approach to the vertex coloring problem', Annals of Operations Research, Vol. 139, pp.229-241.

Kochenberger, G.A., Glover, F., Alidaee, B. and Rego, C. (2004) 'A unified modeling and solution framework for combinatorial optimization problems', OR Spectrum, Vol. 26, No. 2 , pp.237-250.

Laguna, M. and Martí, R. (Eds.) (2003) Scatter Search: Methodology and Implementations in C, Kluwer Academic Publishers, Boston. 
Laguna, M., Martí, R. and Campos, V. (1999) 'Intensification and diversification with elite tabu search solutions for the linear ordering problem', Computers and Operations Research, Vol. 26, pp.1217-1230.

Lewis, M. (2005) A Path-Relinking Multi-Start Tabu Search Metaheuristic for Unconstrained Quadratic Binary Optimization, Hearin Center Technical Report HCES-01-05.

Lewis, M., Alidaee, B. and Kochenberger, G. (2005) 'Using $\mathrm{xQx}$ to model and solve the uncapacitated task allocation problem', Operations Research Letters, Vol. 33, pp.176-182.

LOLIB (1997) Linear Ordering Library, http:/www.iwr.uni-heidelberg.de/iwr/comopt/soft. LOLIB/LOLIB.html

Mitchell, J.E. and Borchers, B. (2000) 'Solving linear ordering problems with a combined interior point/simplex cutting plane algorithm', in Frenk, H. et al. (Eds.): High Performance Optimization, Kluwer Academic Publishers, pp.349-366.

Mitchell, J.E., Pardalos, P.P. and Resende, M.G.C. (1998) 'Interior point methods for combinatorial optimization', in Du, D.Z. and Pardalos, P. (Eds.): Handbook of Combinatorial Optimization, Vol. 1, Kluwer Academic Publishers, pp.189-297.

Palubeckis, G. (2004) 'Multistart tabu search strategies for the unconstrained binary quadratic optimization problem', Annals of Operations Research, Vol. 131, Nos. 1-4, pp.259-282.

Poranen, T. (2005) 'A genetic hillclimbing algorithm for the optimal linear arrangement problem', Fundamenta Informaticae, Vol. 68, No. 4, pp.333-356.

Rao, S. and Richa, A. (2004) 'New approximation techniques for some linear ordering problems'. SIAM J. Computing, Vol. 34, No. 2, pp.388-404.

Steiner, G. and Stephenson, P. (2000) 'Subset-restricted interchange for dynamic min-max scheduling problems', SIAM J. Discrete Math, Vol. 13, No. 4, pp.419-435. 